\title{
Criminal Investigation EIDSS Based on Cooperative Mapping Mechanism
}

\author{
Ping He \\ Department of Information \\ Liaoning Police College \\ Dalian, Liaoning, China
}

\begin{abstract}
On purpose of improving the research in extension intelligence systems when the knowledge in hand is not sufficient, an intuition evidence model (IEM) based on human-computer cooperative is presented. From the initial intuition process space defined by the primitive experience, a series of interactive mapping learning systems (IMLS) with various reductive levels are created. For, each IELS, the rule sets with respective belief degree are induced and saved. The paper introduces cooperative mapping of intuition evidence and object hypothesesmethod to the criminal investigation, and poses a skeleton of cooperative reasoning. The paper views that the reliability of the cooperative reasoning depends on the human-computer interaction results. Simultaneously, choosing the case-cracking clue should be determined by comprehensive evaluations and self-learning of intuition-formal judgments are essentially needed. When applying the model to reasoning and decision making, one can match the intuition judge of the given object to the rule sets of relative nodes, and then draw the conclusion by using some kind of evaluation algorithm. A simple example on how to create and apply the model is give.
\end{abstract}

Keywords-IDSS; EIDSS;intuition evidence; object hypotheses criminal investigation; cooperative reasoning

\section{INTRODUCTION}

Intelligent decision support systems (IDSS) are a method of intelligent system design. From the research achievements of IDSS in recent years, the original intention of researchers is that computers can substitute for the decision-making of human. However, to study the IDSS as an issue in computer science has hampered the system development. No matter in the aspects of knowledge selection, or in uncertain reasoning, though great research achievements have been obtained (especially the introduction of artificial neural network and fuzzy system provides many new tools for development of IDSS), few successful IDSS are available. Many scholars believe that the key to build IDSS is the selection of reasoning model and effective use of knowledge.

Productivity in reasoning model of IDSS is low, reality is complex, and it usually takes a lot of trials to find a satisfactory mathematical description of the phenomenon under consideration. Due to this complexity, modeling has to be done by specialists who are required to speak three "languages": the language of mathematics in which the model is originally described, a programming language or an input-output language to a standard package which is needed to solve the particular case, and the language of the user who is ignorant of these "internal representations", presents his problem in "user- terms" and also needed the relevant features of the model depicted via e.g. graphic means. After all that, the measurement-based model obtained can only be used for the particular situation and has to be adapted for a new application should relevant factors change. In most cases this means redoing the whole identification and estimation process.

In fact, the effective reasoning mechanism of IDSS is cooperative reasoning approach, which combines intuition, knowledge and experience perfectly. However, traditional IDSS do not provide intuitive analysis capabilities for human, but instead rely on scenario evaluation as a means for developing solutions. What kinds of intellectual tasks do we have? Who is more intelligent or smarter: a scientist or a woodmaker (human or machine), a metal-maker or a wood-maker? How to design an IDSS with intuitive learning as the most powerful intellectual function? What is intuition-learning? Can we design computer system with intuitive model? All these topics are subjects of discussion are research hot spots in recent years [1].

In the research and development of Criminal Investigation Intelligent Decision Support Systems (CIIDSS) [2], it has been found that the formation of specific technique and method comes from the intuition and experience of people in dealing with routine duties, and this intuition and experience is nonlinear. In addition, knowledge and common sense are different from each other. Do all problems in reality correspond to some complete knowledge? Experiences in the field for different objects are obviously inconsistent. Accordingly, in the development of applied CIIDSS, the first thing is the selforganization of crime knowledge system and the self-learning of the experience of the crime investigators.

We found that IRMPI of crime analysis is effective tools that they build CIIDSS [3]. In practice, detectives basing on properties resolution of the given case dream up mimetically, and then forming approximate mode image with primary case. Generally case, detective can't see the procedure of the case. Happened, persons can't see that prisoner do all the things on location. Only through mimetic reproduction, we can recognize and master its law of development and changing. So, that it accord with crime character and basic law is rush.

The objective of this paper is to analyze virtual intuitionlearning environments of criminal analysis and to discuss a extension method for criminal investigation IDSS based on the theory of intuition reasoning, That is, Criminal Investigation EIDSS (Extension Intelligent decision support system). 
The rest of this paper is organized as follows: In Section 2 we give a Literature survey about CIIDSS. In Section 3 we describe the overall structure of the intuition learning mechanism. We also discuss in rather more detail the key modules and routines contained in cooperative reasoning. In Section 4 we demonstrate use of the interface in conducting the interactive learning system of criminal investigation. The final section 5 concludes the paper and points out further work.

\section{LITERATURE SURVEY}

\section{A. CIIDSS Based on Knowledge, Intuition and Experience}

As is known, the classical IDSS did not try to build the intuitive model about human brains. But the various reflecting forms of human brains have strong intuitive characteristics [4]. In [Ref. 5], different people show different reflecting degrees. Therefore, the possible space of the intuition of the thing and the various reflecting forms of them in human brains is a multiinput single-output system framework. In the selection of intuition characteristics, for any identification, the reasoning system must rely on the combination of multiple characteristics of the intuition, namely, the traits of an intuition and the existing condition and expansibility of a thing. Intuition reasoning process, composed of elements that are interrelated and mutually restricted, is a complicated phenomenon with multi-factors and layers. Accordingly, intuition concept space should be built from the viewpoint of the constitutive elements.

The literature [6] shows that the criminal investigation work took intuition reasoning as the core. Under the condition that the criminal case has occurred, the investigator frequently applies the intuition experience, makes judgment on basis of the relevant information, and obtains new intuition judgment according to the known intuition concept, so that to further disclose the inside information of the crime. The reasoning mechanism in the IDSS should by no means be alienated from the intuition characteristics of investigation inference. The effective reasoning mechanism is cooperative reasoning approach, which combines intuition, knowledge and experience perfectly. In previous work, the investigators and judger themselves can gain limited direct information via senses.

Sometimes, they can make intuitive judgment according to their own experience without which the investigating work cannot be carried out. In other words, there are full of hypotheses in the investigation of criminal cases. These hypotheses are not imaginations without foundation, but a comprehensive judgment via intuition by combining experience and knowledge. In more occasions, they, especially the judge, must conduct inference on basis of scientific theories and knowledge. The former is called experiential intuitive reasoning, and the latter knowledge reasoning. In the current expert system, the knowledge reasoning method is widely used. But knowledge in certain field is limited, and the reasoning model simply emphasizing knowledge has limitation for many practical problems.

People's experience provides basic intelligence for solve many problems. When the recognitions are different, the basic intelligence is different as well. The tracing to the problem's conditions of the past can propose an experience set. In an artificial system, different people have different behaviors and stories, thus different experiences. Sometimes experiences are called a kind of recognitions; but as the level of recognition is different, the experience of the human is also different. The intelligence of the human is selected and decided by the experience of the human, and the reasonability of the experience's selection is also a meaningful question for discussion.

\section{B. Overview of Reasoning Technique}

In previous work, the investigators and judger themselves can gain limited direct experience via senses. Sometimes, they can make judgment according to automatically generate crime scenarios from the available evidence. In other words, there are full of hypotheses in the investigation of criminal cases. These hypotheses are not imaginations without foundation, but a comprehensive judgment via intuition by combining experience and knowledge. In more occasions, they, especially the judger, must conduct inference on basis of scientific theories and knowledge. The goal of the CIIDSS described is to find the set of hypotheses that follow from crime scenarios that support the entire set of available evidence. This set of hypotheses can be defined as:

$$
\begin{aligned}
& H_{E}=\{h \in H: \exists s \in S,(\forall e \in E, \\
& (S \mapsto e)) \wedge(S \mapsto h)\}
\end{aligned}
$$

where $H_{E}$ is the set of all hypotheses (e.g. accident or murder, or any other important property of a crime scenario) $\mathrm{S}$ is the set of all consistent crime scenarios, our mini-stories in the example $\mathrm{E}$ is the set of all collected pieces of evidence (See figure 1).

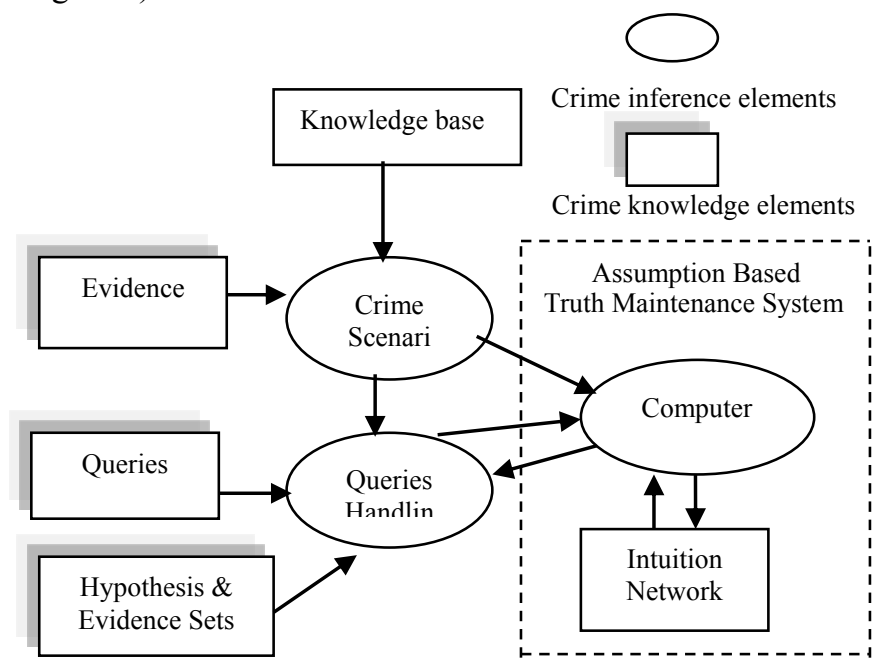

Fig. 1. Basic Architecture of the model based reasoning for crime scenarios.

In establishment of CIIDSS, we have discussed relevant issues of the selection of reasoning technique, that is, cooperative reasoning model [7]. Here, the key is the good combination of human intuition and computer, and what methodology should be adopted to reach the goal of criminal investigation and to fully unfold the trust intuition of the human. [Ref.8] research shows that Intuition Mapping Pattern Inversion (IMPI) is an effective thrust tool to construct this 
intuition behavior. Here, let's first give a hypothesis that, in actual criminal investigation, the investigator conceives a simulation of a specific case by analysis of the case attributes, and constructs an intuitive simulated model approximate to the original case in the brain.

Usually, it is impossible for the investigator to witness the entire process of the case. After the crime, people can experience the scene again, and only by intuitive simulative reconstruction can we learn and grasp its changing patterns. The occurrence of a case gives birth to the latent image of the specific criminal event in an intuition concept space. It is determined by the initial structure of the intuition characteristics of the criminal type. Here, the suspect relationship can be termed as initial image relationship of the brain, and the latent image of the criminal event from the scene is called image relationship. If the image can be determined by the intuition mapping relation, the initial image can be obtained by the image. And this initial image is the suspect of this case. This mechanism is called IMPI of criminal investigation.

\section{The Role of Intuition Learning}

[Ref. 9，2008]describes the Intuition-learning Systems (ILS), also known as, Intuition Learning Networks (ILNs) are online learning venues that emphasize people-to-people (or human to machine) communication combined with traditional and/or intelligence-technology-delivered learning tools. Researchers and practitioners have long been concerned with three fundamental issues involving learning. The first issue involves what people intuition learning, that is, the identifiable knowledge and skills outcomes of learning from accumulated experience. The second issue involves the process of the intuition learning (i.e., just how do we learn?), what are the sequences of events and activities that cause or facilitate learning? The third issue is a more practical one and involves a technology for intuition learning (i.e., designing and building learning environments or learning machines to facilitate the learning process). The fundamental idea behind the concept of a technology for intuition learning is a simulated situation designed to create personal experiences for learners that serve to initiate their own process of inquiry and understanding.

In fact, ILS is a Human-Machine Interaction System (HMIS) based on experience and intuition. What kinds of intellectual tasks do we have? Who is more intelligent or smarter: a scientist or a wood-maker (human or machine), a metal-maker or a wood-maker? How to design a intuition learning system with reasoning as the most powerful intellectual function? What is trusted intuition in learning process? Can we design decision system with trusted intuition? All these topics are subjects of discussion in recent years. The goal of these researches is to find active, productive may be not the best way to determine the starting position and some directions of intelligent learning system design.

At present, most IDSS is designed manually based on past experience of their intuition. Since the number of possible intuition is very large for realistic applications of reasonable complexity, heuristics designed manually may not work well when applied in new problem instances. Further, there is no systematic method to evaluate the effectiveness of IDSS designed manually. For these reasons, an automated method for discovering the proper IDSS for a particular application is very desirable. This leads to the development of our system for automated learning of intuition [10].

\section{CRIMINAL INVESTIGATION EIDSS}

\section{A. Scenario Mapping Intuition Inversion}

Definition 3.1 Let $S=(A, \Gamma, x)$ be a scenario structure system with target original image $x$ of unknown behavior, $S^{*}=\left(A^{*}, \Gamma^{*}, x^{*}\right)$ is a scenario pattern structure system with unknown behavior map. In the reasoning system of IDSS, if there exists a reversible and confirmable behavior scenario mapping $\varphi$, then there exists a scenario mapping intuition inversion from target original behavior fields to scenario behavior fields, it is called scenario mapping and intuition inversion, shorter form scenario mapping intuition inversion(SMII), namely:

$$
(S, x) \stackrel{\varphi}{\longrightarrow}\left(S^{*}, x^{*}\right) \stackrel{\psi}{\longrightarrow} x^{*} \stackrel{\varphi^{-1}}{\longrightarrow} x .
$$

The basic framework of definition 1is shown in Figure 2:

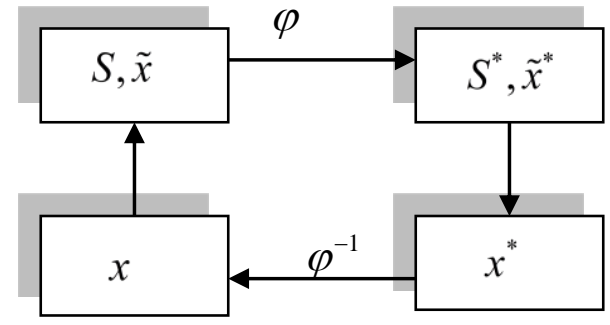

Fig. 2. the Structure of MMII

From Figure 2 it could be discovered that $(S, x)$ expresses a recognition problem with an unknown behavior object $x$, if we can seek out a reversible and shaping $\varphi$ for all such problems. Based on SMII, $x$ can be determined (namely turned unknown $x$ into known $x),(S, x)$ is called SMII resoluble problem for $\varphi$, noted $(S, x ; \varphi)$. If adding the shaping method $\psi$, then the resoluble problem can be expressed as $(S, x ; \varphi, \psi)$. In fact, human brain, for a behavior pattern problem with complexity and uncertainty, undergo limited process of SMII, then form trust discrimination, it is shown in Figure 3:

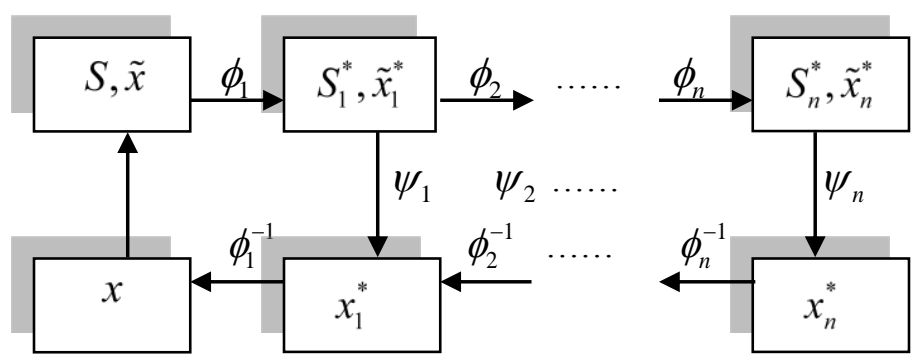

Fig. 3. The study process of SMII 
That is to say, the solution of original image $x$ with unknown behavior pattern is a process with $\mathrm{n}$ scenario mappings $\varphi_{1}, \varphi_{2}, \cdots, \varphi_{n}$ and $\mathrm{n}$ intuition inversion mappings $\varphi_{n}^{-1}, \cdots, \varphi_{2}^{-1}, \varphi_{1}^{-1}$. Where, $\psi$ is a shaping method, which makes sure the map $x_{n}^{*}$ of behavior pattern from scenario map pattern structure $\left(S_{n}^{*}, x_{n}^{*}\right)$. Therefore, the process of this study and recognition is called $\mathrm{n}$ steps SMII process depicted by SMII $^{(n)}$ namely,

$$
\begin{aligned}
& (\mathrm{SMII}) \stackrel{(\mathrm{n})}{:} \\
& (S, x) \stackrel{\phi}{\longrightarrow}\left(S_{n}^{*}, x_{n}^{*}\right) \stackrel{\psi}{\longrightarrow} x_{n}^{*} \stackrel{\phi^{-1}}{\longrightarrow} x .
\end{aligned}
$$

Where $\varphi=\varphi_{n} \varphi_{n-1} \cdots \varphi_{2} \varphi_{1}, \quad \varphi^{-1}=\varphi_{1}^{-1} \varphi_{2}^{-1} \cdots \varphi_{n-1}^{-1} \varphi_{n}^{-1}$.

\section{B. Learning Measurement of Experience-based Intuition}

Definition 3.2 Let $S$ is the objects set of EIDSS, $H$ is the set of all hypotheses, $E(I)$ is the set of all intuition evidence, and there are two mapping, the intuition evidence mapping of the object and the hypotheses mapping of the object based on optimum and non-optimum, then without loss of generality, we have

$$
\begin{aligned}
& D_{E}=\left\{\in S: \exists h \in H\left(\forall e\left(I_{i}\right) \in E(I)\right.\right. \\
& \left.\left(e\left(I_{O}\right) \mapsto s\right) \wedge\left(h_{e} \mapsto s\right)\right\}
\end{aligned}
$$

where $e\left(I_{o}\right)$ (or $e_{o}$ ) is called the evidence feature with trusted intuition, $h_{e}$ is called the hypotheses based on evidence feature.[11] In the research of the intuition learning [6], we introduced the concept of intuition feature index, through which we can describe any evidence factor in the decision making and tell whether it belongs to optimum feature, nonoptimum feature as well as the degree optimum and nonoptimum at same time. According to quantitative expressions, we can give the following definition:

Definition 3.3 Let $S=\left\{s_{1}, s_{2}, \cdots, s_{n}\right\}$ be a objects set of EIDSS, $H=\left\{h, h_{2}, \cdots, h_{n}\right\}$ be a hypotheses set, and there be a set of intuition evidence of optimum in evidence system $E=\left\{e_{1}, \cdots, e_{n}\right\}$, then there be a mapping $e\left(I_{O}\right) \mapsto s$, $h_{e} \mapsto s$ where

$$
\begin{gathered}
e\left(I_{O}\right) \mapsto s: e\left(I_{O}\right) \times S=\left\{\left(e_{i}, s_{i}\right): e_{i} \in E \wedge s_{i} \in S\right\} \\
h_{e} \mapsto s: h_{e} \times S=\left\{\left(h_{e i}, s_{i}\right): h_{e i} \in H \wedge s_{i} \in S\right\}
\end{gathered}
$$

[Ref.4] describes new analysis method of intuitive model, an implementation of our learning strategy. This system can learn high-performance intuitive model for its object application within given resource constraints, and can determine the scope of generality of the learned intuitive model.

\section{Cooperative Reasoning Model}

The auxiliary intuition evidence means the conjunction of the experience information and the similar objects information. Thus, the automatic reasoning system could be founded based on intuition learning in EIDSS. Primarily, the following two mappings are to be founded. If we represent the total collect with $S=\left\{e\left(I_{O}\right), h_{e}\right\}=\left\{s_{1}, s_{2}, \cdots, s_{n}\right\}$, when we input a series of $s_{i} \in S$, we accordingly get the output of the function of the two mappings: evidence-to-make-sure $h_{e}, h_{e i} \in H$. Before making sure the two mappings' characteristics, we should divide the statistical object-evidence groups into trusted object-evidence groups and auxiliary groups. The principle depends on the amount of the information for identifying the evidence-to-make-sure. Let

$$
P\left(s_{i} \mid h_{i}\right)=\frac{N_{\left(s \mid e_{o}\right)}}{N_{(s \mid e)}}
$$

to be the probability of the object $S_{i}$ under the condition $h_{i}$, note: $N_{(s \mid e)}$ is the number of the object choose under the condition of all the evidence, $N_{\left(s \mid e_{o}\right)}$ is the number of the object choose under the condition of trusted the evidence. In addition,

$$
P(S)=\left\{P\left(s_{1}\right), P\left(s_{2}\right), \cdots, P\left(s_{n}\right)\right\}
$$

is the probability distribution of the object choose $S_{i}$. From the detected case database, we can get the relative collect of the intuition evidence and number them. For instance, regarding economical crimes, let the main evidences be $E=\left\{e_{1}, e_{2}, \cdots, e_{n}\right\}$, and then we can go on reasoning following a certain regulations.

For instance, in the case analysis, a certain case has 20 intuition evidences and $h_{e}$ has 15 . With the above method, to calculate how each intuition evidences $e_{i}$ affects a certain hypotheses $h_{e}$ i.e. membership function $\mu\left(h_{e}\right)$, we find the hypotheses choose matrix $M\left(h_{e}\right)$ (row 20, line 15 ), store it into the computer. This is the second type mapping characteristics, give a certain input of this mapping, we get a corresponding $h_{e} \mapsto s$ output. Consider the fact that the number of the intuition evidences $E=\left\{e_{1}, e_{2}, \cdots, e_{20}\right\}$ is large, the trusted hypotheses region is $H=\left\{h_{1}, h_{2}, \cdots, h_{15}\right\}$, when make a hypotheses choose, one couldn't get the details of the 20 evidences simultaneously, but only some of them, i.e. a series of hypotheses $H=\left\{h_{1}, h_{2}, \cdots, h_{15}\right\}$. Thereafter, it's not easy to work out the problem with formulas; 
we change to follow the principle of maximum membership, $\mu\left(h_{e}\right)=\operatorname{Max}\left\{\mu\left(h_{e i}\right), i=1,2, \cdots, 15\right\}$ is the given output and the input of the trusted objects $S_{i}$.

When to recognize a crime, the given information may be not unanimous, so as to computer may find difficult to tell it apart. To solve the problem and enable the computer to reason automatically and find the most valuable intuition evidences, then use the man-computer system to go on with the judgment. Automatic checking function: When input a series of hypotheses $\mu\left(h_{e 1}\right), \cdots \mu\left(h_{e 20}\right)$, if the computer reads

$$
\mu\left(h_{e}\right)=\operatorname{Max}\left\{\mu_{k_{v}}\left(A_{o}\right), j=1,2, \cdots, 20\right\},
$$

let

$$
\varepsilon=\frac{\max \mu\left(h_{e i}\right)-\min \mu\left(h_{e j}\right)}{\mu\left(h_{e}\right)}(i \neq j),
$$

If $\varepsilon<\rho$ should be adjusted, here $\rho$ is the region value, $0<\rho<1$.the affirming method could be as following: Choose a group of cases from the material (the more the better, and not necessarily typical ones, and the intuition evidences are not complete), i.e. each case's evidence $e_{i}$ and each is hypotheses $h_{i}$ known. Input $e_{i}$ to the computer, we can find the corresponding value $\rho(0<\varepsilon<1)$. Choose $\lambda$, for a certain section $\left(\varepsilon_{i}, \varepsilon_{i+\lambda}\right)$, find the statistical number $N_{i}$, and design the total number to be $N_{\left(s \mid e_{o}\right)}$, let

$P\left(\varepsilon_{i}<\varepsilon<\varepsilon_{i+\lambda}\right)=\frac{N_{\left(s \mid e_{o}\right)}}{N_{(s \mid e)}}$

be a corresponding section's $\left(\varepsilon_{i}, \varepsilon_{i+\lambda}\right)$ judging probability, with its distribution we can find the distributions function:

$$
F(\varepsilon)=P\left\{\varepsilon \leq \varepsilon_{i}\right\}=\sum_{\varepsilon \leq \varepsilon_{i}} P\left\{\varepsilon=q_{i}\right\}
$$

Choose the judgment rate $\alpha$, then the threshold value is

$$
F(\varepsilon)=\sum_{\varepsilon \leq \varepsilon_{i}} P\left\{\varepsilon=q_{i}\right\}=\alpha
$$

As a matter of fact, the key of founding the system of fuzzy automatic crime detect reasoning is to make good use of the cooperative reasoning principle. However, it requires good man-computer functions. Meanwhile, the statistical information function is also needed. The studies and practices show that, the using of cooperative reasoning principle combining fuzzy automatic reasoning surely has a promising future. [12]

\section{REALIZATION OF CRIMINAL INVESTIGATION EIDSS}

\section{A. Selection and Establishment of Cooperative Relational Database}

The key to develop the criminal investigation EIDSS is to make it operable, so that to satisfy the practical needs of real criminal investigation. Accordingly, the application of evidence-hypotheses combined with cooperative reasoning in the investigation EIDSS is realized as follows.

Build knowledge base of criminal attributes, relational database of criminal cases and rule base of case solving experience in the computer. These three bases are both independent information sources and interrelated organic whole, which can be called cooperative relational base. Knowledge base of criminal attributes selects all the characteristic expressions of the social crime attributes. It is written into the attribute base in the form of IF AND THEN. Relational database of criminal cases selects the occurrences and solving processes of all criminal cases. It is stored in the computer in the form of data warehouse. Experience rule base selects empirical analysis of various cases and it is stored in the computer in the form of human-computer interaction. [13]

The above discussion reveals that cooperative relationship principle actually accomplishes a kind of reasoning. And the way of this reasoning should conform to the human thinking. Previous researches on AI have made efforts to enable the computer to make decisions like human beings with the assistance of certain algorithms, which has been the goal of research in this field. But the results have been unsatisfying. In the decision making of actual investigation, with certain and limited information, the investigators try to find out the casesolving clues by intuition. Previous works show that intuitive reasoning for decision making is actually a Similarity Inference, which is the repetitive mapping of the nerve stimulus inherent in human brain, and finds the fixed point of the suspect system from judgement of the disordered information by finite self-organization and self-learning. Thus, obtain the ordered objective initial image by self-organizing process of the relationship mapping, that is, build expressions of various relational matrixes, and find the characteristics of criminal attributes from in the scene information, and then find out the range of possibility of the criminal suspect. And determine the criminal suspect according to the additional particular information of the criminal scene. [14]

\section{B. Cooperative Mapping of Crime Investigation EIDSS,}

In establishment of investigation EIDSS, we have discussed relevant issues of the cooperative mapping of intuition evidence and object hypotheses. Here, the key is the good combination of human and computer, and what methodology should be adopted to reach the goal of criminal investigation and to fully unfold the intellectual behavior of the EIDSS. Research shows that cooperative mapping is an effective thrust tool to construct this intellectual behavior. Here, let's first give a set of object hypotheses that, in actual criminal investigation reasoning, the investigator conceives a simulation of a specific case by analysis of the case attributes, and constructs a simulated model approximate to the original case. 
Usually, it is impossible for the investigator to witness the entire process of the case. After the crime, people can experience the scene again, and only by simulative reconstruction can we learn and grasp its changing patterns. The occurrence of a case gives birth to the latent image of the specific criminal event in a certain space. It is determined by the evidence structure of the criminal type. Here, the hypotheses relationship can be termed as evidence relationship, and the latent image of the criminal event from the scene is called image relationship. If the hypotheses can be determined by the two mapping relation, the object can be obtained by the hypotheses. And this crime object is the trusted hypotheses of this case. This mechanism is called extension reasoning of criminal investigation.[15] Abstraction of this principle can be described as follows:

Let $R$ denotes the relationship structure of intuition evidence of a group of criminal object, which includes the criminal object $S_{C}$ to be determined. If $e\left(I_{O}\right) \mapsto s(e)$ denotes a kind of mapping, then the intuition evidence relationship structure $R$ of the criminal behavior can be determined by $e\left(I_{O}\right) \mapsto s(e)$, which, of course, comprises the hypotheses $h_{i}$ of the unknown criminal object $S_{C}$. If hypotheses $h_{i}$ can be decided, then the corresponding $S_{C}$ can be decided by $h_{e} \mapsto s_{c}$. This is the basic framework of EIDSS of criminal investigation (Figure 2).

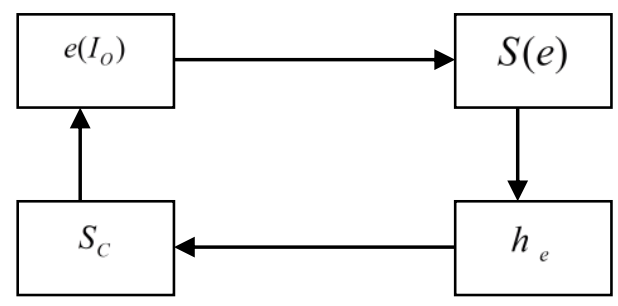

Fig. 4. The basic framework of cooperative mapping

The main construction within system of crime relation lies in case cracking: the intuition evidence and hypotheses -tomake-sure, and can think from evidence to the decision type is the constancy of the two mappings the initial evidence and object mapping.

For real EIDSS, the mapping and inversion have plentiful contents. The cooperative reasoning rule based on criminal investigation knowledge and experience is the product of the combination of human and computer. Its formation process might as well be regarded as mapping accomplished by the human-computer interaction. Thus, the result of cooperative reasoning based on knowledge and experience is the image of the initial image of the suspect system. Inversely mapping the results of cooperative reasoning to the suspect system, the criminal suspect can be determined. This is called mapping inversion process in the expert system.

\section{Criminal Knowledge management Bseed on Intuition Learning}

In order to evaluate the usefulness and the usability of the extended intelligence in a crime case setting, we conducted a study to determine the feasibility of this intelligence analysis tool in real criminal investigations.

First, the general area of the knowledge management of the cooperative mapping has attracted an enormous amount of attention in recent years. Although it has been variously defined, it is evident that knowledge management exists at the enterprise level and is quite distinct from mere intuition learning information. Also apparent in this area are the challenges that knowledge management poses to an organization. In addition to being difficult to manage, knowledge traditionally has been stored on paper or in the minds of people. The cooperative mapping problems facing many firms stem from barriers to access and utilization resulting from the content and format of intuition learning information. These problems make knowledge management acquisition and interpretation a complex and daunting process. Nevertheless, the extension decision technologies of knowledge management have been developed for a number of different applications, such as virtual enterprising, joint ventures, and aerospace engineering.

The same problems of knowledge management exist at the specialized organizations of police department. Many record management systems for crime control agencies contain a large amount of data for each case or incident, but although data may be available, they are not available in a form that makes them useful for higher level processing. A basic task for detectives and crime analysts at Dalian Police Department (DPD) is to create knowledge from cooperation mapping. In this case, the information of cooperative mapping is made up of approximately 1.5 million criminal case reports, containing details from criminal events dating back to 2013. Tacit knowledge has also been described as the means through which new knowledge of cooperative mapping is generated as well as the practical knowledge used to perform a task. It is tacit knowledge that is used as investigators try to tie together information to solve cases and crimes. This ability to combine information to create knowledge is often hampered by the amount of information that exists.

The purpose of this paper is to explore the development of cooperative mapping knowledge system (CMKS) based on intuition learning that can provide the functionality of extension intelligence analysis that currently does not exist in the CMKS. This system is designed to serve as a type of extension knowledge detectives and has been evaluated in a real life context. Its findings also are discussed.

\section{CONCLUSION}

From this pilot study, we conclude that the use of cooperative mapping as a criminal investigation reasoning and intelligence analysis tool in a law enforcement environment is quite promising. 
This paper discusses the critical issues in establishment of EIDSS based on cooperative mapping that should be paid attention to through practice of criminal investigation work. The development of the extension intelligent analysis system must be grounded on identification, otherwise this work is of little significance or value. Simultaneously, the intuitive reasoning should be distinguished from experiential and intuition reasoning. For different cases, experiential reasoning is variable. Only by combining the two together with intuition to reach cooperative mapping can they possibly play their roles in reality. As a matter of fact, the key of founding the system of extension automatic crime detects reasoning is to make good use of the EIDSS. However, it requires good man-computer functions. Meanwhile, the statistical intuition learning function is also needed.

In future work, the method presented here will be expanded upon. Firstly, the representation formalisms employed to describe states and events in intuitive process of criminal investigation will be elaborated. As described earlier, the intuitionistic fuzzy set of states and events that constitute a scenario are restricted by the consistency requirements. This paper introduced a generic means to represent when inconsistencies occur and to prevent inconsistent experience and knowledge from being considered when hypotheses are generated and evidence collection strategies are constructed. When reasoning about related events that take place over experience and intuition, the experience process of the intuition are an important source of such inconsistencies. To avoid overcomplicating this paper, the important issues of knowledge and intuition reasoning were not considered, but will be addressed in future work. Secondly, methods are under development to assess the relative likelihoods of alternative learning system. Several methods to expand the intuition entropy based decision making techniques employed by model based intuitionistic fuzzy diagnosis techniques have been presented in other papers.

\section{ACKNOWLEDGMENTS}

The authors are grateful for the support given by National Natural Science Foundation of China (Grant No. 61272170). We also thank reviewers for insightful and helpful suggestions.

\section{REFERENCES}

[1] Jeroen Keppens, Burkhard Schafer,Knowledge based crime scenario modelling, Expert Systems with Applications, 2006, vol. 30, pp. 203222.

[2] Berestycki, H. and Rodriguez, N. and Ryzhik, L, Traveling Wave Solutions in a Reaction-Diffusion Model for Criminal Activity, submitted for publication, 2013.

[3] He Ping, Tao Weidong, A Design of Criminal Investigation Expert System Based on CILS, Journal of Software, 2011, vol.6, pp1586-1592.

[4] Jin Li, Ping He, Extended automatic reasoning of criminal investigation, IEEE Computer Society, International Conference on Industrial Mechatronics and Automation, 2009, 546-649.

[5] John Mikhail, Moral Grammar and Intuitive Jurisprudence: A Formal Model of Unconscious Moral and Legal Knowledge, Psychology of Learning and Motivation, 2009, vol. 50, pp. 27-100.

[6] He Ping, Design of Interactive Learning System Based on Intuition Concept Space, Journal of computer, 2010, vol.5, pp. 478-487.

[7] Ping He, Crime Knowledge Management Approach Based on Intuition Concept Space, Intelligent Information Technology Application. In: Qihai Zhou, ed, proc. of the Int'l conf IEEE Computer Society, 2008, pp. 276-279.

[8] Giles C. Oatley, Brian W. Ewart, Crimes analysis software: 'pins in maps', clustering and Bayes net prediction, Expert Systems with Applications, 2003,vol. 25, no. 4, pp. 569-588.

[9] He Ping. Fuzzy relationship mapping inversion and automatic reasoning of crime detective, (AIAI2005), Springer, Artificial Intelligence Application and Innovations, 2005, pp.691-700.

[10] F.J. Bex, H. Prakken, and B. Verheij, Anchored narratives in reasoning about evidence, In T. M. van Engers, editor, Legal Knowledge and Information Systems, JURIX: The Nineteenth Annual Conference, IOS Press, 2006, pp.11-20.

[11] S.W. van den Braak and G. Vreeswijk, AVER: Argument visualization for evidential reasoning, In T. M. van Engers, editor, Legal Knowledge and Information Systems, JURIX 2006: The Nineteenth Annual Conference, IOS Press, Amsterdam etc., 2006, pp.151-156.

[12] He Ping, Crime Knowledge Management Based on Intuition Learning System, Fuzzy System and Management Discovery, In:Jun Ma, ed, proc. of the Int'l conf IEEE Computer Society, 2008, pp. 555-559.

[13] Nuno, J.C., Herrero, M.A. and Primicerio, M, A mathematical model of criminal-prone society, Discrete Continuous Dynamical Systems Series $S, 2011$, vol. 4, no. 1, pp. 193-207.

[14] Yung-Chien Sun, Grant Clark.A Computational Model of an Intuitive Reasoner for Ecosystem Control. Expert Systems with Applications, 2009, vol.36, pp. 12529-12536.

[15] Dijkstra, J.J., The influence of an expert system on the user's view: How to fool a lawyer. New Review of Applied Expert Systems, 2006, vol.1, pp.123-138. 